future studies, both epidemiological and clinical trials, used this threshold to create categories or define intervention goals. Thus, while there are a lot of data examining $150 \mathrm{~min} /$ week or more compared with no activity, there are few data examining different doses, intensities, types, or frequencies of exercise. As a result, it is not that $150 \mathrm{~min}$ is the best cut-off point chosen from many doseresponse studies, but rather, it is the cutoff point with the most available data.

Applying the medicine model to exercise, just as one blood pressure drug and one dose are not suitable for all patients, one exercise prescription does not fit all clinical situations. For example, a sedentary, elderly person with multiple chronic conditions is likely to get great benefit from even a small increase in physical activity, such as $60-70 \mathrm{~min} /$ week of low- to moderate-intensity walking, along with some balance and light resistance training. Whereas for a 30-year-old healthy person, 60-70 min is better than nothing, but achieving $150 \mathrm{~min}$ or more a week may have substantially more long-term benefits. The dose and type of physical activity for postmenopausal women to reduce the risk of osteoporosis, is likely to be different from the dose and type of required for weight loss in young people. Important research questions to be examined related to dose and aerobic exercise include the interaction of ethnicity, age, gender and various medical conditions.
Many questions about exercise dose, intensity and type of exercise remain to be answered. For example, we have very few data on doses of resistance or flexibility training that may be beneficial for various outcomes. Resistance, flexibility and balance training have great potential to improve health and function in older adults, and much more research is needed to clarify the relative importance of these activities. Although there are potentially a huge number of permutations to be examined, a few key dose-response studies could produce informative data for the future exercise recommendations.

In summary, while an exercise pill is an exciting concept it is also not likely to be a reality any time soon. However, the nonsense generated by the idea of such an invention creates an opportunity to discuss some real concerns related to physical activity. Central among those is why, given the powerful, pleiotropic medical benefits of exercise, it is not prescribed more in clinical settings? Should this not be an area of investment and focus? Further, there is still a need to refine the exercise prescription. For example, what is the minimal dose of aerobic activity to promote/maintain health and quality of life? How much and what type of resistance training is optimal? Does the prescription vary by age, gender, ethnicity and health status? There is no action (except abstaining from smoking) that could improve health more than being physically active, yet it remains an afterthought in clinical medicine and federal funding priorities. We continue excitably to search for health in a pill, yet we already have the readily available behaviour of regular exercise that would provide enormous benefits. We are missing a great opportunity to prescribe/ promote health at an individual, societal and global level. We call upon professionals in clinical medicine, exercise science and public health to become more aggressive in implementing exercise treatments for all.

Acknowledgements: We thank Peter Katzmarzyk, PhD for his input and review of drafts.

Competing interests: None.

Accepted 18 September 2008

Published Online First 16 October 2008

Br J Sports Med 2009;43:80-81.

doi:10.1136/bjsm.2008.053850

\section{REFERENCES}

1. Narkar V, Downes M, Yu R, et al. AMPK and PPAR agonists are exercise mimetics. Cell 2008;34:405-15.

2. Physical Activity Guidelines Advisory Committee. Physical Activity Guidelines Advisory Committee Report 2008. Washington, DC: US Department of Health and Human Services, 2008.

3. DeVol R, Bedroussian A, Charuworn A, et al. An unhealthy America: the economic burden of chronic disease charting a new course to save lives and increase productivity and economic growth. Milken Institute, October 2007. Available at http://www. milkeninstitute.org/publications/publications. taf?function $=$ detailgID $=38801018 \&$ cat $=$ ResRep (accessed 25 November 2008)

\title{
Too much sitting: a novel and important predictor of chronic disease risk?
}

\author{
N Owen, ${ }^{1}$ A Bauman, ${ }^{2}$ W Brown ${ }^{3}$
}

Research on physical activity and health has pointed clearly to increasing the time that adults spend doing moderate to vigorous intensity activities: 30 minutes

\footnotetext{
${ }^{1}$ School of Population Health, The University of Queensland, Brisbane, Australia; ${ }^{2}$ School of Public Health, The University of Sydney, Sydney, Australia;

${ }^{3}$ School of Human Movement Studies, The University of Queensland, Brisbane, Australia

Correspondence to: Neville Owen, Cancer Prevention Research Centre, School of Population Health, The University of Queensland, Herston Road, Herston 4006, Brisbane, Australia; n.owen@sph.uq.edu.au
}

a day is generally recommended. However, recent evidence underlines the importance of also focusing on sedentary behaviours - the high volumes of time that adults spend sitting in their remaining "non-exercise" waking hours. We provide a brief overview of recent evidence for the distinct relationships between 'too much sitting' and biomarkers of metabolic health and, thus, with increased risk of type 2 diabetes, cardiovascular disease and other prevalent chronic health problems. Particular concerns for this new field include the challenges of changing sedentary behaviours in the context of ubiquitous environmental and social drivers of sitting time; examining the effects of interventions for reducing or breaking-up sitting time and identifying the most relevant implications for clinical and public health practice.

Increasing participation in leisure time physical activity (LTPA) in adult populations is a central tenet of strategies for preventing major chronic diseases (type 2 diabetes, cardiovascular disease, breast and colon cancer) and obesity in developed and developing nations. ${ }^{12}$ To date, clinical practice, community programmes, mass-media campaigns and population strategies have focused mainly on encouraging and supporting individuals to be more active, largely during discretionary or leisure time, but more recently (and to a lesser extent) also in travel time. $^{2}$ While these approaches have met with some success, our recent body of work has identified sedentary behaviour (time spent sitting) as a novel and 
MVPA

$0.7 \mathrm{~h} /$ day

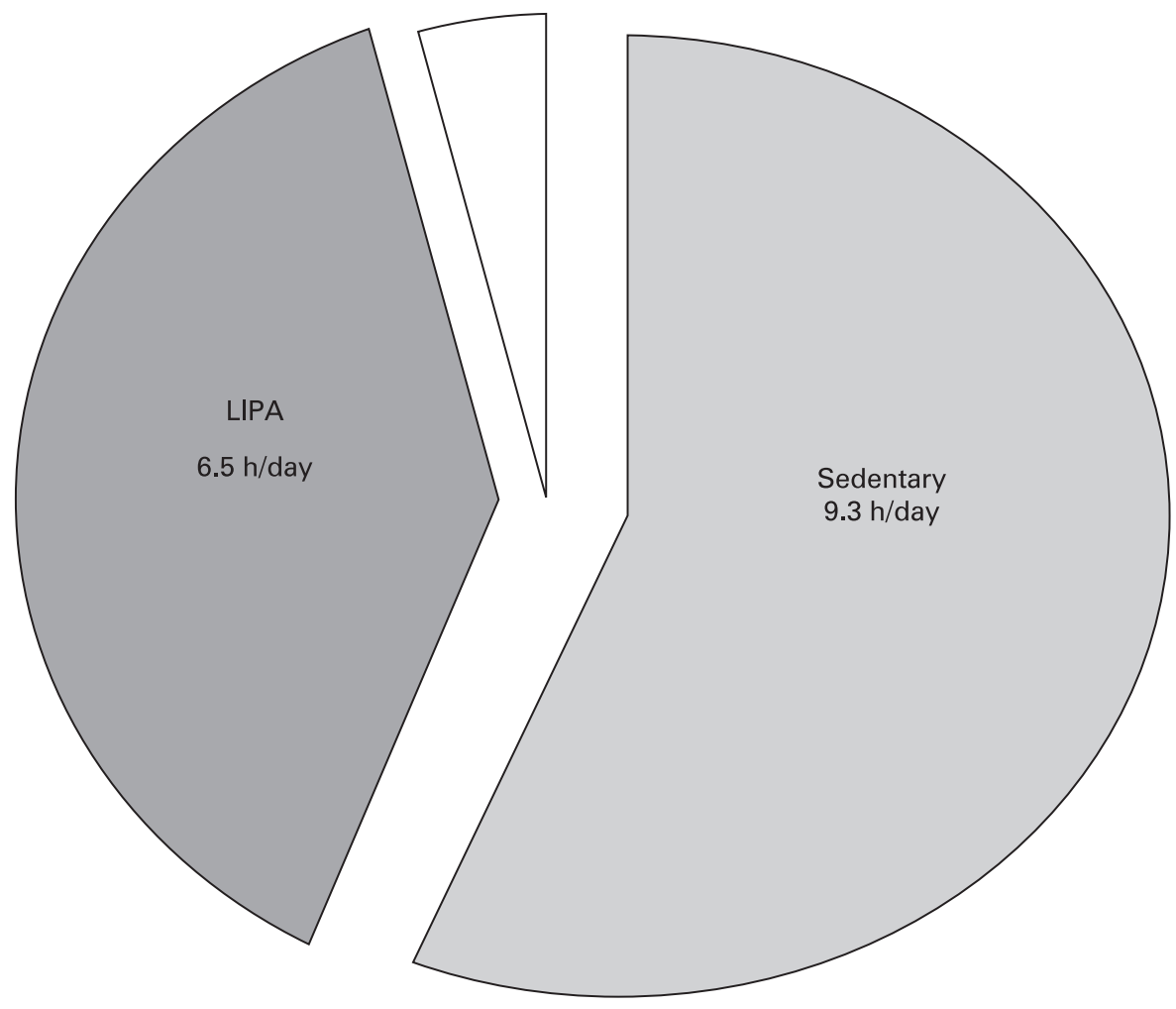

Figure 1 Objectively measured distributions of moderate to vigorous physical activity (MVPA), light intensity physical activity (LIPA) and sedentary time during adults' waking hours.

potentially important risk factor for the development of chronic disease. Even if people meet the current recommendation of 30 minutes of physical activity on most days each week, there may be significant adverse metabolic and health effects from prolonged sitting - the activity that dominates most people's remaining "nonexercise" waking hours.

As population levels of overweight and obesity continue to increase, and chronic health problems from inactivity become increasingly prevalent, there is now an imperative to increase population levels of overall total daily energy expenditure. However, discretionary participation in moderate to vigorous physical activity is insufficient to raise energy expenditure as much as is necessary for population-level obesity prevention. ${ }^{3}$ Objective data derived from a recent accelerometer study, ${ }^{4}$ in which physical activity was measured objectively (as opposed to being self-reported), show that adults, on average, spend more than half their waking hours in sedentary activities (primarily prolonged sitting). The remainder of the time is spent in light intensity physical activity (LIPA; predominantly standing with some ambulation) and only about $4-5 \%$ of the day is spent in moderate to vigorous physical activity (MVPA) (fig 1). ${ }^{4}$ It is clear that if sedentary time decreases, then time spent in LIPA or MVPA will increase. While the metabolic and health consequences of actual shifts in sedentary time relative to LIPA and MVPA are currently unknown, new evidence now suggests that such challenges need to be addressed.

Compelling recent evidence emphasises the need to influence sedentary behaviour - to limit excessive sitting - and to reduce its likely health consequences. This will involve very different approaches from those needed to increase LTPA, such as exercise prescription. The behaviour of sitting time occurs in different contexts, including sitting for transport, at work, at home and in leisure time. It also requires that researchers determine the quantum of the increase in total physical activity (LIPA or MVPA) that would be required to achieve better health outcomes and prevent obesity and identify how best to go about achieving such changes.

\section{TOO MUCH SITTING-THE HEALTH CONSEQUENCES OF SEDENTARY BEHAVIOUR}

New evidence suggests that contemporary changes in transport, occupations, domestic tasks and leisure activities have had negative effects on daily energy expenditure. ${ }^{5-8}$ Sedentary behaviour (from the Latin sedere - "to sit") is the term now used to characterise those behaviours for which energy expenditure is low, including prolonged sitting time in transit, at work, at home and in leisure time. In this context, the metabolic equivalent (MET) is used to define body mass neutral energy expenditure of activities, as the ratio of the metabolic rate of the activity and resting metabolic rate, which is defined as 1 MET. ${ }^{9}$ Running has a MET value of at least 8 METS, moderate-pace walking has a value of about 3-4 METs and sedentary behaviours are in the range of 1-1.5 METs. ${ }^{10} 11$

A recently published prospective study showed that self-reported sitting time (as a marker of sedentary behaviour) was a predictor of weight gain in Australian women, even after adjustment for energy intake and leisure time physical activity. ${ }^{11}$ This was followed by observational studies using objective measurement of sedentary time, which showed that not only is total sedentary time important for blood glucose control but also that a larger number of breaks in sedentary time are associated with more favourable metabolic profiles. ${ }^{4}{ }^{12}$ Additionally, these relationships have been found to be consistently stronger for women than for men. ${ }^{11}{ }^{12}$

Recent findings also suggest that leisure time MVPA, in the context of otherwise sedentary lifestyles (fig 1), ${ }^{4}$ is unlikely to be sufficient to prevent increasing population levels of overweight, obesity and chronic disease. ${ }^{3}$

More than two-thirds of the mid-age population in developed countries like the UK, the USA and Australia is now overweight or obese, which poses additional significant health risks for this generation. ${ }^{13}$ Also, about $6 \%$ of children are also now obese $\mathrm{e}^{14}$ and this obesity is likely to track into adulthood..$^{15}$ Both overweight/ obesity and physical inactivity in mid-age are strong markers for the development of non-communicable disease over time. ${ }^{16}{ }^{17}$ Thus, reducing sitting time may have at least as important a role as promoting physical activity in maintaining healthy weight and in preventing further weight gain and improving chronic disease outcomes in mid-age adults; it is crucial for the future health of ageing populations.

What is now needed is a broader, innovative approach to understanding and influencing sedentary behaviour in addition to increasing physical activity. This requires a paradigm shift, so that one 
thinks about the balance of sedentary behaviour and activity in all aspects of daily life, including transport, occupational settings, domestic work and leisure, especially around obesity management and the role of total physical activity in the prevention of further weight gain. This involves some re-orienting of the physical activity and health field from its well-established focus on LTPA to a comprehensive programme of research to understand the determinants of sedentary behaviour and both LIPA and MVPA. It will also involve studying the effects on health outcomes of shifting the balance of these behaviours towards more activity in all domains of daily life.

\section{CLINICAL IMPLICATIONS}

Given the recent recognition of this phenomenon of too much sitting, there are not yet any recommended clinical guidelines. Commonsense might suggest that it may be prudent to try to minimise prolonged sitting with 5 minute breaks every hour. However, more specific advice will require dose-response relationships between sitting and health outcomes to be defined using controlled studies. Many of the possible interventions that encourage movement may well be undertaken in settings in which prolonged periods of sedentary behaviours are the norm.

\section{CONCLUSIONS}

Research, policy and practice on physical activity and population health has focussed largely on increasing the time that adults spend doing moderate to vigorous intensity activities; 30 minutes a day is generally the target. However, recent evidence from biomarker studies and objective-measurement studies (and also from some prospective epidemiological studies) highlights the importance of focusing on the balance of light-intensity activities and sedentary behaviours-particularly the high volumes of time that adults in industrialised and developing countries spend sitting in their 15.5 "nonexercise" waking hours. A particular concern for this new research agenda is how to approach reducing or breaking-up prolonged sitting time, and how this might relate to increasing light intensity and moderate to vigorous intensity physical activities. Other research opportunities include carrying out studies on how best to promote higher volumes of overall physical activity (light intensity activities in addition to moderate to vigorous intensity activities), in the context of the ubiquitous environmental and social drivers of sitting time in occupational, transportation, recreational and domestic settings.

Particular concerns for exercise science research agenda include identifying why sedentary behaviour and the associated health relationships seem to be particularly strong for women and examining the effects of interventions for reducing or breaking-up sitting time. The issue of too much sitting has challenging implications for future healthcare practice and will require development of new kinds of clinical and public health guidelines. ${ }^{18}$

Funding: $N O, A B$ WB are supported by NHMRC Program Grant funding (\#301200); NO is also supported by a Queensland Health Core Research Infrastructure grant.

\section{Competing interests: None.}

Accepted 24 October 2008

Published Online First 28 November 2008

Br J Sports Med 2009;43:81-83.

doi:10.1136/bjsm.2008.055269

\section{REFERENCES}

1. Daar AS, Singer PA, Persad DL, et al. Grand challenges in chronic non-communicable diseases. Nature 2007; 450:494-6.

2. Bauman A, Bellew B, Vita P, et al. Getting Australia active: Best practice for the promotion of physical activity. Melbourne: National Public Health Partnership, 2002.

3. Bauman A, Allman-Farinelli M, Huxley $\mathrm{R}$, et al. Leisure-time physical activity alone may not be a sufficient public health approach to prevent obesitya focus on China. Obes Rev 2008;9:119-26.

4. Healy GN, Dunstan DW, Salmon J, et al. Objectively measured light-intensity physical activity is independently associated with 2-h plasma glucose. Diabetes Care 2007;30:1384-9.

5. Anderssen SA, Engeland A, Sogaard AJ, et al. Changes in physical activity behavior and the development of body mass index during the last 30 years in Norway. Scand J Med Sci Sports 2008;18:309-17.

6. Levine JA. Nonexercise activity thermogenesisliberating the life-force. J Intern Med 2007;262:273-87.

7. Bell AC, Ge K, Popkin BM. The road to obesity or the path to prevention: motorized transportation and obesity in China. Obes Res 2002;10:277-83.

8. Brown W, Ringuet $\mathrm{C}$, Trost $\mathrm{S}$. How active are young adult women? Health Promot J Austr 2002;13:23-38.

9. Ainsworth BE, Haskell WL, Whitt MC, et al. Compendium of physical activities: an update of activity codes and MET intensities. Med Sci Sports Exerc 2000;32:S498-504.

10. Gunn SM, Brooks AG, Withers RT, et al. Determining energy expenditure during some household and garden tasks. Med Sci Sports Exerc 2002;34:895-902.

11. Brown WJ, Williams L, Ford JH, et al. Identifying the energy gap: magnitude and determinants of 5 -year weight gain in midage women. Obes Res 2005; 13:1431-41.

12. Healy GN, Dunstan DW, Salmon J, et al. Breaks in sedentary time: beneficial associations with metabolic risk. Diabetes Care 2008;31:661-6.

13. Booth ML, Chey T, Wake M, et al. Change in the prevalence of overweight and obesity among young Australians, 1969-1997. Am J Clin Nutr 2003;77:2936 .

14. Australian Institute for Health and Welfare. Are all Australians gaining weight? Differentials in overweight and obesity among adults, 1989-90 to 2001. Bulletin No. 11. AlHW Cat. No. AUS 39. Canberra: AlHW, 2003.

15. Venn AJ, Thomson RJ, Schmidt MD, et al. Overweight and obesity from childhood to adulthood: a follow-up of participants in the 1985 Australian Schools Health and Fitness Survey. Med J Aust 2007:186:458-60.

16. Hu FB, Willett WC, Li T, et al. Adiposity as compared with physical activity in predicting mortality among women. N Engl J Med 2004;351:2694-703.

17. Brown WJ, Hockey R, Dobson A. Rose revisited: a "middle road" prevention strategy to reduce noncommunicable chronic disease risk. Bull World Health Organ 2007:85:886-7.

18. Hamilton M, Healy G, Dunstan D, et al. Too little exercise and too much sitting: Inactivity physiology and the need for new recommendations on sedentary behaviour. Current Cardiovascular Risk Reports 2008;2:292-8.

\section{Is the measurement of maximal oxygen intake passé?}

\section{Roy J Shephard}

A recent and controversial review ${ }^{1}$ suggests that the measurement of maximal

Correspondence to: Roy J Shephard, PO Box 521, Brackendale, Canada BC VON 1H0; royjshep@shaw.ca oxygen intake is passé. The author concludes (p. 554) "It is now time to develop novel testing methods.... That the measured VO2max is a relatively poor predictor of both the performance potential of athletes with similar athletic ability and of the changes in performance that occur with continued training should encourage both basic and applied sports scientists to reconsider the real value of this iconic test."

A number of the arguments that are advanced in this review seem to need correction or refutation. Specifically, this riposte will examine whether a maximal treadmill test is an unrealistic procedure for athletes, whether a unimodal approach to testing is appropriate in sports medicine, and whether an alternative laboratory test 